\section{A RESPONSE TO "THE POTENTIAL LONG-TERM COMPARATIVE EFFECTIVENESS OF LAROTRECTINIB AND ENTRECTINIB FOR SECOND-LINE TREATMENT OF TRK FUSION- POSITIVE METASTATIC LUNG CANCER"}

We read with intrigue the recent publication in this journal by Roth et al. (2020) "The Potential LongTerm Comparative Effectiveness of Larotrectinib and Entrectinib for Second-Line Treatment of TRK FusionPositive Metastatic Lung Cancer."1 It was surprising to see that this manuscript was published, since there are several major methodological limitations of the analysis that prevent the application of these findings to health care decisions. These analyses do not support the authors' strong conclusions in favor of larotrectinib and, therefore, could mislead health care providers.

\section{Extrapolation of within-trial survival curves from very small patient subgroups with limited follow-up}

The comparison used in the study is based on a subset of 12 and 10 patients with TRK fusion-positive metastatic non-small-cell lung cancer (NSCLC) from the larotrectinib and entrectinib trials, ${ }^{2,3}$ respectively. The data were derived after only 13 months of follow-up in both trials and extrapolated to generate lifetime survival curves. While the authors had complete information for larotrectinib, progression-free and overall survival curves for entrectinib in this subpopulation have not been published, so the authors made assumptions about these distributions. These limitations impart a high level of uncertainty to all estimates.
2. Naive, unadjusted, cross-trial comparison based on single-arm studies with clearly different patient populations

When head-to-head trials of 2 therapies are not available, network meta-analyses/indirect treatment comparison methods may be used to inform decisions, if the analyses can be anchored to a common control arm. The ISPOR Good Practice Task Force guidance for network meta-analyses/ indirect treatment comparisons considers naive comparisons between 2 trials to be a "fatal flaw" in the analysis. ${ }^{4}$ In some cases, comparisons between therapies across singlearm studies may be considered using newer methods, such as simulated treatment comparison and matching-adjusted indirect comparison. While they may allow adjustment for differences in patient characteristics to some extent, even these techniques have limitations when the sample sizes are small and there is high heterogeneity in patient characteristics between the 2 trials. Chu et al. (2020), in their systematic review of the efficacy of TRK inhibitors, state that "a robust quantitative synthesis of results comparing the relative efficacy of the different NTRK mutation-specific agents versus the current standards of care is challenging." 5

Despite evidence of substantial differences between the larotrectinib and entrectinib single-arm trials, the authors conducted a naive comparative effectiveness analysis, with no statistical adjustment to account for these differences. The source used by the authors for baseline characteristics of patients with NSCLC in larotrectinib trials is unclear, since the reference (Hyman et al. ${ }^{2}$ ) cited by the authors does not present data for the subset of patients with NSCLC. Data for the subset of patients with NSCLC in the larotrectinib trials are presented in the publication by Farago et al. (2019), ${ }^{6}$ which we cite here.

The following key differences in the trial populations at baseline highlight that the entrectinib trial population was older, with a higher proportion of patients with central nervous system (CNS) metastases and poorer Eastern Cooperative Oncology Group performance status (ECOG PS) than that of the larotrectinib trial population:

- Age: Patients in the larotrectinib trial (median age 49 years; range 25-76) ${ }^{6}$ were much younger than patients in the entrectinib trial (median age 62 years; range 46-76). ${ }^{3}$ In fact, the youngest patient in the larotrectinib trial was 21 years younger than the youngest patient in the entrectinib trial.

- CNS metastases at baseline: A higher proportion of patients in the entrectinib trial (60\%) had brain metastases at baseline compared with the larotrectinib trial (50\%). ${ }^{3,6}$

- ECOG PS: While Farago et al. did not provide ECOG PS data for larotrectinib, these data were given in the Drilon et al. (2019). presentation, which included 11 of the 12 patients with NSCLC. ${ }^{6,7}$ A greater proportion of patients in the entrectinib trial had an ECOG PS of 2 (20\%) compared with larotrectinib (9\%).

\section{Cost of larotrectinib compared} with entrectinib

Based on the wholesale acquisition cost (March 6, 2020), the monthly cost for larotrectinib $(\$ 33,281)$ is almost twice that for entrectinib $(\$ 17,046)$.

While the Roth et al. analysis evaluates quality-adjusted life-years regardless of the limitations, the authors chose not to consider the relative costs of the 2 therapies despite the substantial difference, which is surprising given the authors' health economics expertise.

\section{Summary}

Given the heterogeneity resulting from the very different, small patient subpopulations and lack of statistical adjustment, the analyses presented by Roth et al. should be interpreted with caution. We appreciate that the authors conducted sensitivity analyses using worst- and best-case 
scenarios; however, these do not overcome the inherent limitations of single-arm studies with vastly different patient characteristics. The authors' conclusion that "larotrectinib provided improved life-year and quality-adjusted life-years outcomes compared to entrectinib" is an inappropriate extrapolation using a limited dataset and does not provide robust information to the readers.

\author{
Sarika Ogale, PhD \\ Genentech \\ South Francisco, CA \\ ogale.sarika@gene.com \\ Clarissa Emy Higuchi Zerbini, BBA \\ F. Hoffmann-La Roche \\ Basel, Switzerland \\ Miranta Antoniou, PhD \\ F. Hoffmann-La Roche \\ Basel, Switzerland \\ Romain Freund, MD \\ F. Hoffmann-La Roche \\ Basel, Switzerland
}

\section{DISCLOSURES}

The writing of this letter was sponsored by Roche/Genentech. All authors are employees of, and hold stocks in, F. Hoffmann-La Roche Ltd/Genentech Inc.

\section{REFERENCES}

1. Roth JA, Carlson JJ, Xia F, Williamson T, Sullivan SD. The potential long-term comparative effectiveness of larotrectinib and entrectinib for second-line treatment of TRK fusion-positive metastatic lung cancer. J Manag Care Spec Pharm. 2020;26(8):981-86. doi: 10.18553/ jmcp.2020.20045

2. Hyman DM, van Tilburg CM, Albert CM, et al. Durability of response with larotrectinib in adult and pediatric patients with TRK fusion cancer. Ann Oncol. 2019;30(Suppl 5):v159-93 (Abstract 445PD).
3. Paz-Ares L, Doebele RC, Farago AF, et al. Entrectinib in NTRK fusion-positive nonsmall cell lung cancer (NSCLC): integrated analysis of patients (pts) enrolled in STARTRK-2, STARTRK-1 and ALKA-372001. Ann Oncol. 2019;30(Suppl. 2):ii48-49 (Abstract 1130).

4. Hoaglin DC, Hawkins N, Jansen JP, et al. Conducting indirect-treatmentcomparison and network-meta-analysis studies: report of the ISPOR Task Force on Indirect Treatment Comparisons Good Research Practices: part 2. Value Health. 2011;14(4):429-37.

5. Chu P, Batson S, Hodgson M, Mitchell CR, Steenrod A. Systematic review of neurotrophic tropomyosin-related kinase inhibition as a tumor-agnostic management strategy. Future Oncol. 2020;16(4):61-74.

6. Farago A, Kummar S, Moreno V, et al. Activity of larotrectinib in TRK fusion lung cancer. J Thorac Oncol. 2019;14(10):S28384 (Abstract MA09.07).

7. Drilon AE, DuBois SG, Farago AF, et al. Activity of larotrectinib in TRK fusion cancer patients with brain metastases or primary central nervous system tumors. J Clin Oncol. 2019;37(15 Suppl):Abstract 2006.

\section{THE AUTHORS RESPOND}

We thank Ogale et al. from Roche/ Genentech for their interest in our recent publication "The Potential Long-Term Comparative Effectiveness of Larotrectinib and Entrectinib for Second-Line Treatment of TRK FusionPositive Metastatic Lung Cancer."1 They note many valid limitations of preliminary simulation modeling to understanding the potential comparative effectiveness of emerging therapies, most of which were discussed in our original publication. The new issues raised by Ogale et al. can be grouped into 2 main points, which are addressed as follows.

First, Ogale et al. suggest that it is problematic to publish preliminary simulation-based comparative effectiveness assessments that extrapolate data from small samples. It is important to note that our publication in JMCP was categorized as a "Brief Report"-a category of manuscript that "is reserved for small or pilot studies that have limited generalizability or descriptive studies that may not test a hypothesis or have comparative study groups."2 We believe that our publication is well aligned with the scope of that category.

Furthermore, Ogale et al. note alternative approaches to assess comparative effectiveness but do not critically review their feasibility. One approach that was suggested is network meta-analysis, but it is unclear to us how such an analysis would be conducted when larotrectinib and entrectinib have only been evaluated in single-arm phase $1 / 2$ trials. Additionally, the authors suggest matching adjusted indirect comparison as an alternative analytic approach, but such matching would yield limited benefit in larotrectinib and entrectinib non-small cell lung cancer (NSCLC) samples of 12 and 10, respectively. ${ }^{1}$

Exploring the shortcomings of these alternative study designs help to highlight the rationale and need for the preliminary comparative effectiveness modeling study that we conducted. Nonetheless, there are many limitations to this approach, which we discuss in our publication. Our study is intended to provide initial insights into the potential comparative effectiveness of larotrectinib versus entrectinib until higher levels of evidence can be developed.

Second, Ogale et al. characterize the interpretation of our study results as "strong," present an inaccurate quote to support this assertion (the underlined text in the following excerpt is omitted), and omit qualifying language before and after the quote that does not support the 